\title{
Estado de desarrollo de la archivística clásica hasta los años 30 del siglo XX: Tres manuales archivísticos de trascendencia universal
}

\author{
Leomar José Montilla Peña \\ Universidad Politécnica Territorial Andrés Eloy Blanco - UPTAEB, Venezuela
}

Mayra M. Mena Mujica

Universidad de la Habana - UH, Cuba

\section{ANÁLISIS}

\begin{abstract}
Resumen
La finalidad de este artículo es presentar una revisión teórica del estado desarrollado de la archivística clásica hasta las tres primeras décadas del siglo XX, con el propósito de exponer las teorías y las referencias de la sujeción epistemológica de esta ciencia, es decir, describir los presupuestos del discurso científico técnico en la que orbita esta disciplina científica. Para poder desarrollar algunas ideas preliminares sobre este discernimiento plantearemos primero una panorámica general de los tres primeros manuales publicados y sus autores dentro del contexto europeo. Después, nos referiremos a la caracterización de la archivística concebida hasta ese periodo describiendo brevemente el objeto de estudio, los principios y conceptos fundamentales y los procesos de tratamiento de los documentos de archivo.
\end{abstract}

Palabras clave

Ciencia Archivística ; Archivística clásica ; Muller, Samuel ; Feith, Johan ; Fruin, Robert ; Jenkinson, Hilary ; Casanova, Eugenio

\section{Development Status of classical archivistics to the 30s of XX century: Three archivistics manuals of universal significance}

\begin{abstract}
The purpose of this article is to present a theoretical review the developed state the of classical archivistics until the first three decades of the XX century, with the purpose of exposing the theories and references of the epistemological subjection of this science, that is, describe the budgets the technical scientific discourse in which orbits this scientific discipline. In order to develop some preliminary ideas on this discernment will raise first an overview of the first three manuals published and their authors within the European context. Then, we refer to the characterization of archival conceived to the period briefly describing the object study, the principles and fundamental concepts and processes treatment of document archival.
\end{abstract}

Keywords

Archival Science ; Classical archivistics ; Muller, Samuel ; Feith, Johan ; Fruin, Robert ; Jenkinson, Hilary ; Casanova, Eugenio 


\section{Introducción}

El origen de la escritura permitió al hombre comunicar y registrar sus actos en soportes perdurables pues, como apunta Duranti (1993) "...la necesidad de memoria surge naturalmente en cualquier organización” (p. 29, traducida por los autores) como consecuencia de la:

...necesidad que tienen los creadores de documentos de llevar a cabo sus actividades por medio del registro de esa información, en documentos en los cuáles ellos puedan confiar y la necesidad de la sociedad de preservar tales documentos para recordar, mantenerse, protegerse y perpetuarse a sí misma." (Duranti, 1992, p. 50, traducida por los autores).

El volumen y la complejidad de tales actividades humanas, ya desde el mundo antiguo, condujeron al establecimiento de los primeros archivos conocidos en la historia de la humanidad. El surgimiento de estos, consecuentemente, condicionó la aparición de un conjunto de técnicas para ordenar y conservar la información generada por los individuos o las instituciones de acuerdo a sus atribuciones. La evolución y el desarrollo de estas técnicas, unidas a circunstancias político sociales específicas como la revolución francesa de 1789, propiciaron y condujeron a la aparición, a mediados del siglo XIX en el continente europeo, de la llamada Archivística. Desde entonces, y en diversos espacios geográficos, han aparecido diversas formulaciones teórico-prácticas en la búsqueda de un cuerpo doctrinal para esta ciencia, que a la vez ha permitido solventar los problemas producidos por los procedimientos burocráticos y administrativos de las instituciones de acuerdo a sus estructuras sociales, políticas, económicas, culturales y tecnológicas.

Numerosos autores han propuesto periodizaciones que permiten comprender el desarrollo evolutivo de la Archivística como ciencia. Sin embargo, a pesar de tal diversidad, existe consenso en el campo de esta ciencia respecto a que hay un primer período en su desarrollo que va desde mediados del siglo XIX hasta la década de los 80 del siglo XX. Este período ha sido calificado por diferentes autores como la etapa estatista o dominante (Cook, 1997), clásica (Thomassen, 1999) o histórico-tecnicista (Riveiro, 2001) de la Archivística. Para los propósitos de esta investigación, por obvia necesidad de síntesis, en lo adelante adoptaremos el calificativo de clásica para referirnos al estadio de desarrollo de esta ciencia hacia el año1930, periodo en que se confina los tres manuales más trascendentales para la organización de los documentos de archivo.

Puede considerarse que la evolución de las técnicas empíricas de tratamiento de los documentos, desarrolladas desde la más remota antigüedad, hasta el surgimiento de la Archivística como ciencia para la "administración de los archivos", en el siglo XIX, se debe a que las instituciones gubernamentales en el contexto europeo entendieron que los archivos ya no podían seguir considerándose solo como un "repositorio" de documentos "históricos", porque a ellos también llegaban de forma "más o menos regular" documentos generados como consecuencia del registro de la información de los actos de las diferentes instituciones de los estados y que era necesaria su organización para su uso posterior (Duchein, 1992, p. 18, traducido por los autores). Así, según Moreno (2004) “...los nacionalismos germinados venidos de la mano del romanticismo ideológico o la historiografía positivista prestarán singulares sustratos metodológicos a la archivística." (p. 85).

\section{Los autores de la construcción archivística clásica desde 1898 hasta 1930}

Puede considerarse que la línea de desarrollo de las ideas en el campo de la Archivística hasta 1930, está claramente marcada por las obras de los holandeses Müller, Feith y Fruin, el inglés Hilary Jenkinson y el italiano Eugenio Casanova. 


\subsection{El trío holandés, pioneros en el campo de la archivística}

En 1898 se da la luz a lo que se considera la primera obra científica del campo de la Archivística, el Manual de Organización y Descripción de Archivos de los holandeses Samuel Müller (1848-1922), Johan Feith (1858-1913) y Robert Fruin (1857-1935); quienes por primera vez sistematizaron en esta obra las aportaciones teóricas en torno al concepto de principio de procedencia y orden natural de los documentos propuestas, desde 1841, por autores franceses y alemanes. El trío holandés, como también se les conoce, consolidó en esta obra los fundamentos teóricos, metodológicos y técnicos para la organización de los archivos, dando a la Archivística su originario estatuto científico. El primero de ellos Müller tenía formación de historiador y los otros dos, Feith y Fruin, eran abogados. Sin embargo, se consagraron como eminentes archivistas por sus publicaciones para la renovación de los archivos, ejerciendo toda su vida como archiveros estadales, al tiempo que fueron fundadores, integrantes y directores de la Sociedad Holandesa de Archivistas (en adelante VAN). (Biografisch Woordenboek van Nederland, 2011, traducido por los autores).

La publicación del Manual fue el resultado de un encargo de la VAN al triunvirato para que trabajaran en las directrices para la organización y descripción de los documentos en los archivos holandeses, en colaboración entre el Ministerio de Interior y los Archivos del Estado de los Países Bajos. Una fuente importante para la elaboración del Manual fueron los debates teórico prácticos aparecidos en la revista Archievenblad, que había sido fundada por la propia VAN en 1892. También sirvieron de punto de partida al Manual el reglamento de los archivos estadales holandeses, publicado en 1897, y las aportaciones de autores alemanes, franceses e ingleses relacionados con la Diplomática, el principio de procedencia y orden natural de los documentos y la noción de custodia. (Horsman, Ketelaar \& Thomassen, 2003, p.254-258, traducido por los autores).

El Manual vio la luz en mayo de 1898, en la ciudad de Haarlem, bajo la denominación de Handleiding voor het ordenen em beschrijving van Archieve, y estructurada en forma de parágrafos contó con seis capítulos, en los que precedidos por una introducción, se exponían un centenar de "reglas". En el primer capítulo se aborda todo lo relacionado con el origen y la composición de los depósitos de archivos, el segundo se ocupa de la disposición de los documentos dentro del archivo, el tercero trata sobre los elementos para la descripción de los documentos, el cuarto está dedicado a la elaboración de inventarios, en el capítulo quinto se esbozan las instrucciones adicionales para la descripción y el sexto se referiré al uso convencional de ciertos términos y signos. (Muller, Feith, \& Fruin, 2003, traducido por los autores).

Esta obra les valió a sus autores un gran reconocimiento a nivel mundial, y aún hoy es considerada una referencia fundamental y necesaria en el campo de la archivística. Esta ha propiciado las numerosas traducciones del Manual a diferentes idiomas, como los casos del alemán en 1905, el italiano en 1908, el francés, 1910 , el inglés 1940 y portugués en 1960." (Neue Deutsche Biographie, 2010, traducido por los autores). Como se puede observar, lamentablemente el Manual Holandés aún no cuenta con una publicación en idioma castellano.

\subsection{Manual de Hilary Jenkinson}

Como hemos apuntado otra de las voces claves de la Archivística clásica es la de Sir Charles Hilary Jenkinson (1882-1961). Graduado de la Universidad de Cambridge y tras sus estudios de griego y latín, Jenkinson consagró su vida al trabajo como archivero en el Archivo Público del Reino Unido; fue asesor del programa para tiempo de guerra del ejército británico, ejerció la enseñanza de la archivística en la Universidad de Londres, fue miembro destacado de la Asociación Británica de Archiveros y se desempeñó como secretario y presidente del Public Records Offices. (Wedgeworth, 1993, p. 412-414, traducido por los autores).

En 1922 Jenkinson publicó su Manual of Archive Administration, que estructurado en cinco partes, es considerado por Cook (1997) “...el segundo tratado más importante de la teoría y la práctica de la archivística.” (p. 22-23, traducido por los autores). El Manual cuenta con una primera parte dedicada a una introducción general, en la segunda parte describe el origen y desarrollo de archivos y las normas para guardar documentos, la tercera parte teoriza y conceptualiza sobre los archivos modernos, las nuevas tendencias y lo que Jenkinson denomina la regla de oro para la transformación del archivo; en la cuarta describe los métodos y mecanismos de producción, registro y 
distribución de los documentos y en la parte final aborda los archivos de la primera guerra mundial de acuerdo con su propia experiencia en la guerra. (Jekinson, 1922, traducido por los autores).

Jekinson plasmó en cada una de las partes del manual sus hallazgos y experiencias de trabajo con los archivos medievales, las instituciones del poder público y sus vínculos con el entorno social, así como los mecanismos de restauración administrativa. Sin embargo, una de las aportaciones que aún hoy vuelve las miradas de archiveros y estudios de la Archivística al Manual de Jenkinson es la introducción del concepto de evidencia, así como del término "evidencialidad", para referir los vínculos inextricables entre el documento y su contexto de surgimiento y su frase "la santidad de la evidencia", incluida en el texto del Manual, pasó a ser punto de partida para posteriores desarrollos teóricos.

Otra de las aportaciones claves del Manual jenkinsoniano fue su consideración de que los archivos son espacios informativos originados por una actividad administrativa para satisfacer necesidades informativas, y que su ordenación, en consecuencia, se debe basar en el respeto a su acumulación natural y no en una artificial basada en criterios externos a ese orden.

\subsection{La archivistica de Casanova}

Seis años después de la publicación del Manual de Jenkinson el italiano Eugenio Casanova (1867- 1951) publicaba su Archivistica. Casanova, abogado y archivista de profesión, trabajo en los Archivos del Estado de Siena, Florencia, Turín y Nápoles, en este último obtuvo el nombramiento de director del Archivo Grande. También fungió como director de los Archivos Estadales y del Archivo del Reino de Roma. Sus enfoques y aportaciones teóricas lo consagran como uno de los archivistas pioneros de la Archivística. Se destacó también en el campo de la enseñanza de la Archivística, especialmente con su trabajo durante años en la Universidad de Roma. Esgrimió como idea esencial la necesidad de una Archivística independiente y autónoma despojada de su condición de técnica auxiliar para los historiadores y diplomatistas. (Dizionario Biografico degli Italiani, 2011, traducido por los autores).

Casanova, estructuró su obra en cinco capítulos. Aunque en lo que puede considerarse una introducción a la obra hace un análisis general del archivo y la Archivística. En el primer capítulo Casanova describe todo lo relacionado con los edificios, construcción, distribución de espacio, mantenimiento, seguridad, entre otros aspectos de los edificios propios para archivos. En el segundo se refiere al tratamiento archivístico de los documentos. El tercer capítulo explica la fuente de los archivos y la Archivística y en el cuarto capítulo aborda la naturaleza jurídica y uso del archivo. Este último capítulo se divide en dos partes, una primera él aborda la legislación y reglamentación archivística y en la segunda la comunicación de los documentos. (Casanova, 1928, traducido por los autores).

Otro aspecto importante en la obra de Casanova es que aportó el primer intento de periodización del desarrollo de los archivos y de las prácticas archivísticas realizadas desde la antigüedad. Casanova propone una periodización basada en cuatro grandes períodos. El primero se inicia en el mundo antiguo hasta el siglo XIII, el segundo lo comprenden los siglos XIII hasta el XV, entre estos periodos el archivero italiano deja ver claramente el carácter conservador de los documentos jurídicos que daban fe de las posesiones que tenían los hombres y los gobiernos, por ello consideraban a los archivos como patrimonio cultural. La tercera parte abarca desde los siglos XVI al XVIII, en esta se manifiesta que el archivo fue considerado como el repositorio documental para la construcción de la historia. El último período de la propuesta de Casanova lo comprende el siglo XIX y hasta el la segunda década del siglo XX, en que según su consideración empieza a observar la Archivística como ciencia independiente, que se fundamentaba en el principio de procedencia y orden natural de los documentos, que él llamaba el método histórico, es decir un método basado en la historia del órgano creador de los documentos. (Ibídem., p. 291-442, traducido por los autores).

Según Oddo Bucci Casanova le "...dio a la disciplina su enfoque empírico, la construyó como una ciencia descriptiva y le aplicó la obligación de la historiografía positivista, la cual estaba dirigida a la acumulación de los hechos en vez de la elaboración de conceptos..." (Oddo Bucci, 1992, p. 11, citado en Cook, Ob. Cit., p. 9, traducido por los autores). 
Son estos tres autores y sin dudas sus principales obras las que nos permitirán caracterizar a la Archivística desarrollada hasta 1930.

\section{Caracterización de la archivística clásica hasta 1930}

Intentar caracterizar el estadio de desarrollo de la Archivística hasta 1930 para el momento de la publicación de las tres obras más transcendente, nos obliga a acercarnos a tres aspectos que consideramos esenciales para cumplir tal propósito: el objeto de estudio de la Archivística en este período, sus formulaciones teorías y principios fundamentales y los procesos de tratamiento de los documentos.

Ahora bien antes de adentrarnos en el análisis de los tres aspectos apuntados resulta necesario precisar que la comprensión del estadio de desarrollo de la Archivística para 1930 es heredera de acontecimientos históricos, socio políticos y culturales ocurridos en Europa durante y después de la Revolución Francesa de 1789, e inspirados por la propia revolución. Durante todo el siglo XVIII se había producido en Europa una descomunal concentración de los archivos en grandes depósitos que las monarquías utilizaron de forma intensiva y abusiva, casi exclusivamente al servicio de sus propias políticas. Los revolucionarios franceses en respuesta a esto y en su afán de implementar en toda su magnitud su lema de igualdad, libertar y fraternidad para todos los ciudadanos, tomaron medidas respecto a sus archivos que afectan directamente el nacimiento, desarrollo y evolución de la Archivística como ciencia, pues como apunta Malheiro (2002):

\footnotetext{
...el golpe al antiguo régimen pasaba inevitablemente también por los archivos. Allí se conservaban los fundamentos de la organización del Estado, los registros de las deliberaciones más odiadas por los revolucionarios, los títulos de nobleza y de las propiedades de los partidarios de la monarquía. Además de eso los gobernantes salidos de la revolución sentían que era necesario crear un nuevo servicio de archivo que se encargase de velar por los documentos oficiales en los que en el pasado se había asentado el viejo régimen. (p. 100, traducido por los autores)
}

En consecuencia dos son las decisiones de los revolucionarios franceses que afectan a los archivos:

1. La creación de los Archivos Nacionales como repositorio general central para la custodia de las fuentes de la historia nacional de todo el territorio francés, decretado por la Convención Francesa el 18 Brumario del Año II. Primer archivo histórico en la historia de la humanidad.

2. La proclamación del principio de publicidad de los archivos y su declaración como propiedad nacional, permitiendo el acceso libre de los ciudadanos. Decreto la Convención de de 25 de junio de 1794.

Estas decisiones van a tener un impacto importante en la aparición de la nueva Archivística, pues como apunta Duranti (1993) “...los documentos creados antes de la Revolución y no necesarios ya para la administración del nuevo Estado, fueron concentrados en el Archivo Nacional [...] y abiertos a la consulta, mientras que los documentos que permanecían activos fueron mantenidos en las oficinas por la nueva administración y permanecieron en secreto. Esto determinó una material y teórico distinción entre archivos administrativos e históricos..." (p. 29, traducido por los autores).

Malheiro (Ob. Cit.) resume este hecho considerando que: 
... comienzan aquí a distinguirse dos tipos de acervos: el indispensable para la administración y aquel que, no siéndole ya necesario, sólo conserva un mero interés histórico - cultural [...] así se quiebra la unidad estructural de los archivos (existente desde la antigüedad) y se produce una desarticulación metódica de los acervos de origen, todo en nombre de valores extraños al criterio que presidió su concentración por parte de las entidades productoras. Se golpeaba así un modelo milenario cuya razón de ser se asentaba en el respeto por la red de relaciones entre los documentos que integraban un mismo sistema de información. (p.101, traducido por los autores).

Las ideas de los revolucionarios franceses respecto a los archivos tuvieron mucho éxito en el resto de Europa y fueron poco a poco determinando el panorama de los archivos en todo el viejo continente, incluso en aquellos países como el Reino Unido que no tuvieron incidencia directa de la impronta napoleónica. Es consecuencia, los archivos históricos se extendieron por toda Europa y se convirtieron en el archivo por excelencia en estas sociedades.

Por otra parte en las primeras décadas del siglo XIX se asiste a una profunda renovación de las técnicas historiográficas, inspiradas por “...el desarrollo del positivismo [...] que preconiza la verificación documental (análisis de evidencias debidamente representadas) como método al servicio de la historia" (Malheiro, Ob. Cit. p. 108, traducida por los autores). Estos desarrollos, en consecuencia, refuerzan al documento de archivo como fuente objetiva de información para la validación de los resultados de investigación de la historia positivista.

Es en este escenario, el de la aparición de los archivos históricos en toda Europa y el del uso del documento archivístico, casi exclusivamente, como fuente objetiva al servicio investigación histórica, que comienzan a aparecer las primeras formulaciones teóricas que conducen a la aparición, en la segunda mitad del siglo XIX, de la Archivística como ciencia.

\subsection{El objeto de estudio de la archivística clásica}

Cualquier acercamiento a la delimitación del objeto de estudio de la Archivística clásica nos conduce necesariamente a su definición en el ya mencionado Manual de los holandeses. Muller, Feith and Fruin identifican, según Thomanssen (1999), al objeto de estudio de la Archivística como “... el conjunto de documentos creados o recibidos por una administración o una oficina, cuya entidad básica es el documento físico y en donde las interrelaciones entre estos son consideradas orgánicas y naturales” (párr. 5, traducido por los autores). Como se puede apreciar la definición de objeto de la Archivística está circunscrita a los archivos, ya que estos son: “...el conjunto de documentos escritos recibidos o producidos oficialmente por determinado órgano administrativo o por uno de sus funcionarios, en la medida en que tales documentos se destinaban a permanecer en la custodia de ese órgano o de ese funcionario" (Muller et al., Ob. Cit., p. 13, traducida por los autores).

Idea similar aparece en el concepto de archivo, como objeto de la Archivística que encontramos en la obra de Jenkinson (Ob. Cit.) cuando define que archivo es "el conjunto de documentos de cualquier naturaleza, que todo cuerpo administrativo, toda persona física o moral reúne de modo automática y orgánicamente, por razón misma natural o jurídica de sus funciones o de sus actividades" (p. 41, traducido por los autores).

Similar concepción sobre el objeto de la Archivística aparece en la obra de Casanova (Ob. Cit.), pues define que "El archivo es la colección ordenada de los actos de una institución, formada en el curso de su actividad y preservada para la consecución de fines políticos, jurídicos y culturales de esa entidad” (p. 19, traducido por los autores).

Aunque puede considerarse que en Casanova hay una intensión sistémica e integradora entre archivos administrativos e históricos, e incluso privados, que lamentablemente no se hizo dominante en la teoría de la Archivística clásica. En tal sentido Malheiro (Ob. Cit.) apunta que Casanova “... no separa archivo administrativo (registratura o ufficio di protocolo) de histórico (archivo) - dado que el segundo deriva del primero -, en un mismo concepto integra tanto archivos públicos como privados" (p. 127, traducido por los autores). Esta última idea sobre los archivos privados es explicada por Borja de Aguinagalde cuando apunta: 


\begin{abstract}
...Casanova distingue entre Archivos Privados y "carteggi" (o correspondencias) y plantea precisamente el problema en unos términos que no han sido desafortunadamente desarrollados con posterioridad por otras investigaciones. Términos de extraordinario interés: Casanova plantea la cuestión (...) de la cesión al Estado por parte del individuo (el ciudadano) de parcelas de responsabilidad, segmentos de su propia memoria, además de la facultad de velar por la correcta conservación y gestión de la misma. (Tascini, 1991, p.271, traducido por los autores).
\end{abstract}

Ahora bien, quizá la mejor asimilación de las ideas de estos tres clásicos respecto al objeto de estudio de la Archivística Clásica podemos encontrarla en diccionarios y obras posteriores a estos clásicos. Por solo poner dos ejemplos, el Diccionario de Terminología del Consejo Internacional de Archivos (1984) define Archivística como la "disciplina que trata de los aspectos teóricos y prácticos de los archivos y de su función." En tanto que Heredia (1991) la define como "...la ciencia de los archivos (...) que estudia la naturaleza de los archivos, los principios de su conservación y organización y los medios para su utilización” (p. 29).

Es importante aclarar que en todas estas definiciones el término Archivo es visto desde el punto de vista del Archivo Históricos, pues la Archivística se concentró en la nueva función histórico cultural de los archivos, desatendiendo durante siglos aquella otra dimensión genética de los archivos, los archivos administrativos. Una de las causas de esta situación es, como apuntamos arriba, la importancia que dieron los historiadores al documento archivístico como fuente casi única para la investigación. Esto hizo que la Archivística se centrara exclusivamente en ofrecer un conjunto de técnicas que garantizaran el acceso y uso de documentos antiguos y medievales a los historiadores.

Un aspecto importante relacionado con el objeto de estudio de la Archivística clásica, que permite caracterizarla, es la supremacía que se da en este período a los documentos emanados de las administraciones como los objetos por excelencia de los archivos históricos. Como se puede apreciar en las definiciones de los tres autores clásicos todos hacen referencia a que el contenido de los archivos proviene de un "órgano administrativo" (trio holandés), "cuerpo administrativo" (Jenkinson), "institución" (Casanova). En tal sentido Cook (Ob. Cit.) es muy esclarecedor cuando apunta que "...la experiencia que estos autores tenían se circunscribía a un número limitado de documentos medievales, susceptible del cuidadoso análisis diplomático, o con los documentos emanados de los bien organizados registros departamentales dentro de las administraciones" (p.6, traducida por los autores). Este enfoque de la Archivística clásica casi exclusivamente en documentos emanados de las administraciones le valió el calificativo de Archivística "Estatista" o del Estado. (Ibídem, p. 16, traducido por los autores).

Otro elemento importante que caracteriza a la Archivística clásica, y que puede deducirse de las definiciones aportadas por los clásicos, es que esta se concentra en la preservación y tratamiento de objetos informativos físicos. En tal sentido es muy ilustrativa la referencia a documentos "escritos a manos, dibujados o impresos" hecha por el trío holandés cuando se refieren al Archivo como objeto de la Archivística. También nos deja ver este elemento de "lidia" de la Archivística clásica con elementos físico, el hecho de que con frecuencia muchos autores introducen en el propio concepto de archivo "...los edificios, las instalaciones por cuanto tienen que ver con la conservación de aquéllos (se refiere a los documentos)". (Heredia, Ob. Cit. p. 31).

Como apuntamos arriba, a través de Thomassen, otro elemento sustantivo del objeto de la Archivística clásica está en el hecho de que los conjuntos de documentos que forman el Archivo tienen una génesis "natural", es decir, los documentos se originan de las acciones "de una persona física o jurídica, un ente, una oficina" y no intencionadamente por el impulso de un individuo o un colectivo para "crear artificialmente un archivo" (Gutiérrez, Lodolini, Tanodi \& Gallego, 1991, p. 33). En tal sentido y en el origen de practica archivística, los archivos se distinguieron como "Lugar (físico) de preservación de los documentos bajo la jurisdicción de una autoridad pública. El lugar (físico) que dotaba a los documentos de veracidad, les concedía la capacidad de servir como evidencia y memoria continua de las acciones." (Duranti, 1995, p. 243, traducida por los autores). 
Todos estos presupuestos son resumidos de forma magistral por Fuster Ruiz (1999) cuando define a la Archivística como:

\begin{abstract}
...la ciencia que se ocupa de los archivos en sus aspectos teóricos y prácticos, estableciendo principios inalterables y estudiando técnicas adecuadas de gestión de documentos, administración y tratamiento técnico de archivos, así como la función jurídica, administrativa y científica de los mismos, desde un punto de vista archivístico o de ciencias y técnicas diversas, y su relación con las entidades productoras de los conjuntos orgánicos de documentos, a fin de manejar y hacer accesible la información de los fondos documentales. (p. 117).
\end{abstract}

Por otra parte, Theo Thomassen (Ob. Cit.) precisa que el objeto de estudio de la archivística clásica tiene dos dimensiones:

Desde el punto de vista Diplomático su objeto fue un diploma simple. Desde el punto de vista de la administración fueron los fondos. Pero, al menos en los países bajos, el fondo fue visto como el conjunto de documentos no corrientes de una comunidad, ciudad, provincia, estado, etc. y no como los documentos corrientes de los diferentes cuerpos administrativos de esa comunidad. La entidad básica fue, desde el punto de vista de la diplomática, el diploma simple o registro, pero desde el punto de vista administrativo este fue el ítem individual. (párr. 17, traducido por los autores).

\title{
3.2. Principios y conceptos fundamentales de la archivística clásica
}

\section{a) El principio de procedencia y orden natural de los documentos}

Cook (Ob. Cit.) aclara que "...la principal contribución de los autores holandeses fue articular los principios (o "reglas") más importantes concernientes tanto a la naturaleza como al tratamiento de los archivos..." y que de "...forma sencilla están los conceptos de procedencia y el orden original. (p. 25, traducido por los autores).

Ahora bien, según Martín Pozuelo-Campillo (1996) "la idea fundamental de la teoría holandesa se expone en los parágrafos uno, dos y dieciséis." del Manual de 1898. En la regla número uno, como ya apuntamos, los holandeses enuncian su concepto de archivo como conjunto de documentos, aunque precisan que "...esta definición será la base sobre la que se levantará todo demás." (p. 33).

En la segunda regla expresan que "...un archivo es un todo orgánico..." (Muller et al, Ob. Cit., p. 19, traducido por los autores), aunque aclaran que este "...no se crea arbitrariamente, sino que crece, se forma y sufre transformaciones según reglas fijas.” (Martín-Pozuelo, Ob. Cit., p. 33).

Ahora bien, es en la regla dieciséis donde los holandeses sintetizan todas sus ideas respecto al principio de procedencia y orden natural de los documentos, pues afirman que los documentos generados por un creador deben conservarse separados cuidadosamente y no ser mezclados con los archivos de otros creadores, y que "El sistema de clasificación de los documentos de un archivo debe estar basado en la organización original del archivo, a la que en esencia corresponde la organización del órgano administrativo que lo produjo" (Muller et al, Ob. Cit., p. 52, traducido por los autores). Esta regla es sin dudas la más importante para los holandeses, pues establece en esencia un método para la organización de los archivos históricos que por primera vez no estaba basada en presupuestos ajenos a la naturaleza de los archivos, como habían sido las clasificaciones artificiales cronológicas, geográficas o de materias introducidas en los archivos por influencia de los historiadores. En tal sentido los holandeses sentenciaron que "Para organización del archivo (...), urge antes que nada, restablecer cuanto sea posible el orden original. Solamente entonces será posible juzgar conveniente (...) apartarse de él." (Ibídem).

Es importante hacer notar como el aspecto físico nuevamente aparece en las formulaciones teóricas de la Archivística clásica, esta vez respecto al principio de procedencia y orden natural de los documentos. 
Nótese como para los holandeses este orden emana de un principio físico, es decir, la procedencia de los documentos es el lugar, la oficina que los produce, dentro del "órgano administrativo".

\section{b. El concepto de evidencia}

Otro de los conceptos que sin duda resultan claves para la comprensión del estadio de desarrollo de la Archivística para 1930 es el de evidencia. Este concepto fue introducido por Jenkinson en su ya mencionado Manual de 1922, desde la consideración de que la cualquier importancia que tengan los documentos de archivo está relacionada con el hecho de estos son evidencias de los actos o transacciones de sus creadores.

Jenkinson, asumiendo la metodología para el tratamiento de los archivos y el enfoque de los conceptos de procedencia y orden natural del Manual de los holandés, afirma que la salvaguarda de la evidencia de los documentos está relacionada con preservar la posición y la relación natural que estos muestran en el momento en que llegan al archivo, la posición y la relación natural establecidas por el creador de los documentos. Mantener la evidencia significaba, entonces, la conservación de la estructura del contexto documental de cada documento. Así para Jenkinson la evidencia es producto de las necesidades operacionales del creador, sólo él debe determinarla y a él se le asigna la tarea se establece la selección de aquellos documentos que deberán ser preservados para el largo plazo.

Ahora bien, para Jenkinson, desde una clara postura positivista, el concepto de evidencia estaba acompañado de dos adjetivos que la calificaban, imparcial e impoluta, pues el hecho de que los documentos fueran subproducto natural del registro de las actividades de los creadores y por tanto emanación orgánica de estos mismos creadores. En tal sentido es esta evidencia imparcial e impoluta la que convierte a los archivos y a los documentos atesorados en ellos fuentes objetivas para la investigación.

Esta perspectiva del concepto de evidencia da al archivista el papel de mero conservado de los documentos, es decir, su papel es el de salvaguardar de aquella evidencia incluida en los documentos. Así Jenkinson (Ob. Cit.) afirma rotundamente en varias de sus obras que:

\footnotetext{
La carrera del archivista es una carrera de servicio. Ellos existen para facilitar el trabajo de otras personas. Su credo, la santidad de la evidencia; su tarea, la conservación de cada pedazo de evidencia adherido al documento con el que trabajan; su objetivo brindar, sin prejuicio para todos los que deseen conocer, los medios del conocimiento. (p. 190, traducido por los autores)
}

Son estos conceptos, procedencia y orden natural y evidencia, junto al concepto de archivo, explicado en el acápite anterior, los que articulan y estructuran el aparato teórico de la Archivística clásica y condicionan, obviamente, los procesos de tratamiento de los documentos.

\subsection{Los procesos de tratamiento de los documentos en la archivística clásica}

Puede considerarse que los procesos sustanciales de la Archivística hasta 1930 son los de ordenación y clasificación y valoración de los documentos archivísticos.

\section{a. Ordenación y clasificación de los documentos}

Los presupuestos de la ordenación y clasificación de los documentos en la Archivística clásica son los enunciados en el Manual de los holandeses respecto al principio de procedencia, ya apuntado. Es importante recalcar que el trío holandés sistematizó el conjunto de ideas aparecidas en Europa, desde mediados del siglo XIX, respecto a la procedencia y que estas ideas fueron el resultado de un profundo 
diálogo crítico de los autores que las defendían frente a propuestas de clasificación de tipo cronológico, materia y geográficas entronizadas en el ámbito de la clasificación de archivos por influencia de la metodología de la Historia y por los nuevos intereses culturales con que se asumieron los archivos después de la revolución francesa de 1789. Estas clasificaciones son calificadas en el Manual Holandés como "anti naturales".

Podemos afirmar entonces que el punto de partida para la organización y clasificación de los documentos de archivo en este período es la ya mencionada regla dieciséis del manual de los holandeses, es decir, el principio de procedencia y el orden natural de los documentos. Este principio marca los pasos para la organización de los fondos y su observación permite establecer su clasificación y la ordenación. Los autores explican este proceso de la siguiente forma:

Dos Sistemas se considerarán si se puede. El primero adopta diferentes cabeceras arbitrariamente establecidas (por ejemplo, el Cuerpo Administrativo, Finanzas, Servicios, etc.), Correspondiente a esas reglas, que suelen aparecer en los catálogos de la biblioteca. El otro sistema, por el contrario, no establece ninguna cabecera arbitraria, sino sólo aquellos que son sugeridos por el carácter y la organización de las series del archivo en sí, es decir, los encabezados de conformidad con las diversas ramas de la autoridad administrativa que producen la serie de archivo. (Muller et al, Ob. Cit., p. 52, traducido por los autores).

Los holandeses describen que existen hasta ese momento dos métodos para la ordenación y clasificación de los archivos y que lo que los distingue es la arbitrariedad del primero, pues este establece arbitrariamente ciertas cabeceras, conduciendo a una clasificación artificial. Es segundo método resulta en clasificaciones naturales, pues:

La organización original de un archivo debe coincidir, por supuesto, con las líneas principales de la vieja estructura de la entidad administrativa que lo produjo, no hace falta decirlo. Esa vieja organización no fue formada arbitrariamente, no es el resultado de la casualidad, sino que es la consecuencia lógica de la forma en que se constituye la unidad administrativa, cuyos deberes del archivo es el resultado. Este cuerpo construido, por así decirlo, su archivo, y al hacerlo, se tuvieron en cuenta las necesidades de su propia constitución. (Muller et al, Ob. Cit., p. 56 y 57, traducido por los autores).

Estas reglas subyacen en la comprensión de que el método de ordenación y clasificación se base en el respeto la estructura orgánica de la institución. Esta afirmación se encuentra recogida en la regla número dieciséis del Manual holandés, como apuntamos, y también en las reglas diecisiete y dieciocho de la siguiente manera:

...el orden original debe primero que todo hallarse restablecido en la medida de lo posible. Sólo así se puede juzgar después, y en qué extensión, es deseable desviarse de ese orden (Muller et al, Ob. Cit., p. 60 y 61, traducido por los autores). Más adelante dice, se puede apreciar la base fundamental para la ordenación que permitirá determinar la organización del archivo: La originaria ordenación de un archivo puede ser cambiada para corregir las desviaciones de la constitución general del archivo, tanto si derivan de errores de las direcciones generales del archivo como si provienen de cambios introducidos en el sistema de custodia de los documentos archivísticos. (Muller et al, Ob. Cit., p. 62, traducido por los autores). 
Entre la propuestas de estas reglas los holandeses también determinaron que para el método de organización de las series, en la cual todo documento se lleva a un grupo de archivo, se tenían que establecer las secciones en los archivos de acuerdo con la del grupo a la que pertenecía el documento, afirmación se recoge en la regla veintiuno de la forma siguiente: "No es el objeto de un documento, pero su destino que debe determinar el lugar que va a ocupar en la colección de archivo." (Muller et al, Ob. Cit., p. 72, traducido por los autores)

Lo interesante de estas fundamentaciones es que demuestran la importancia de la estructura administrativa para la organización, y que de esta se puede determinar la clasificación de acuerdo al orden original y el respecto de los fondos de las instituciones que los producen, considerando sus características estructurales donde el archivero siempre ha de considerar el orden original, y que su uso es determinante para organizar los documentos producidos y agrupados de acuerdo a la forma seriada de las secciones, series y expediente. Reconstruyendo la estructura del fondo documental donde los documentos serán ordenados en cada serie de manera consecutiva, de acuerdo ha como se dieron las actuaciones administrativas que proporcionaron su origen de producción.

Martín Pozuelo-Campillos (Ob. Cit.), argumenta que:

De esta forma debemos considerar el tratamiento y la organización original de los documentos desde la propia institución u organismo productor de aquellos como el punto de partida del tratamiento y la organización posterior de los mismos desde el archivo. Lo cual en absoluto significa mantener un desorden, si éste fue en origen del estado en que se encontraron aquellos documentos, sino que más propiamente se trata de mantener el estado natural y originario de aquellos. Entendiendo por estado natural el que deriva de su propia naturaleza de origen, esto es, la disposición de los documentos cuando éstos se preparaban para cumplir un cometido concreto y cuando lo cumplían finalmente. El posterior desorden de los mismos nada nos dice de su función originaría, sino que más bien se deriva del hecho de que la final consecución de aquella función pudo muy fácilmente generar un abandono de los propios documentos que propició el desorden de los mismos. Así el respeto a la estructura original de los fondos y al orden natural de los documentos debe entenderse desde la singular concreción del respeto a su estado natural originario, esto es en el caso de documentos singulares, su vinculación a la función social que lo produjo, en el caso de documentos complejos, expedientes. (p. 149).

Theo Thomassen (Ob. Cit.), manifiesta que los argumentos de los holandeses son uno de los más importantes en la consolidación de la Archivística clásica ya que tiene: que ver con qué:

El acercamiento diplomático demandó una investigación completa de todos los diplomas emitidos alguna vez y su exhaustiva descripción y -de ser posible- su publicación crítica en orden cronológico. El acercamiento administrativo demandó la descripción de todas las series e ítems de los fondos, vistos como el todo de los documentos no corrientes de la comunidad, organizados de acuerdo al principio de respecto de los fondos y el Provenienz-prinzip (el principio de procedencia), aplicado a todo el conjunto de los documentos no corrientes de tal comunidad. (párr.20).

Las ideas de Jenkinson (Ob. Cit.) respecto a la ordenación y clasificación de los documentos de archivo en general coinciden con las planteadas en el Manual holandés, pues considera que el proceso de organización del archivo tiene que partir del principio de procedencia, es decir: “...debemos dejar en claro que la única base correcta para la ordenación es la exposición de los objetos que originalmente sirvieron a los Archivos Administrativos." (p. 80, traducida por los autores). 
Así Jenkinson declara que el proceso para la organización del archivo es responsabilidad del archivero y que el procedimiento de esta actividad se da “... en dos partes: el primero, el estudio de la administración concretamente, su historia y su organización, la segunda, dividiendo los Archivos en clases, la subdivisión de éstas, y la subdivisiones nuevas (...) El más importante de todos los principios de la Gestión de Archivos se llama el respeto a los fondos." (Ibídem, p. 84, traducida por los autores).

Para complementar la clasificación, Jekinson establece como ineludible realizar un inventario de las clases y las subclases de los fondos para ayudar en la confección de las herramientas descriptivas de los grupos de archivos "Vamos a suponer, entonces, que la realización, de manera temporal, todo el trabajo esencial de los Archivos", donde el archivero debe "producir un trabajo hacia satisfacer las necesidades especiales de los investigadores". Es decir, elaborar instrumentos operativos para la investigación, estableciendo como primer requisito "las guías" donde se realiza la descripción de los fondos generales o grupos de archivos, los plazos y la disposición de los documentos, seguidamente "los índices y repertorios descriptivos" de los documentos custodiados en los grupos de archivos, que se hallan en las "clases o series de archivos" para facilitar el acceso a los documentos y por último un "Calendario" provisto como un documento informativo sobre los documentos contenido de los grupos de archivos a través de una listado ordenado para poner una descripción total o parcial de cuáles serán conservados como verdades impolutas." (Ibídem, p. 108 110, traducida por los autores).

El aporte de Casanova a la clasificación es situado por Lodolini en el hecho de "Eugenio Casanova examinó los archivos familiares y personales bajo el aspecto de la "Archivística pura" o la teoría archivística, dando las normas para su clasificación." (Tascini, Ob. Cit., p. 54, Traducido por los autores)

Eugenio Casanova (Ob. Cit.) también reconoce que para la organización del archivo se debe considerar el principio de procedencia y el orden natural de los documentos y que para realizar la clasificación es necesario:

\begin{abstract}
Distinguir con precisión y asignar definitivamente a que materia o servicio va remitir el asunto o práctica que viene: regístralo y numerarlo para que con mayor facilidad se pueda rastrear, es la operación más delicada y fundamental por el registro, a saber, la clasificación de documentos. (p. 141, traducido por los autores)
\end{abstract}

Casanova enfatiza en la necesidad de implementar símbolos para el ordenamiento de los documentos y que esto perimiese su ubicación de manera más efectiva en el fondo, inclusive describe y ejemplifica sobre los modelos de ordenamientos alfabético, cronológico, decimal y de materia. Respecto al uso del método decimal considera que los archiveros pueden valerse del método propuesto por Melvin Dewey para las bibliotecas, pero dejando claro que estos símbolos solo eran compresivos para los libros y que eran de pluralidad sobre contenido de estos y que ese caso no se podría aplicar en la clasificación en los archivos por que el contenido de los documentos de archivo no coincide "casi nunca", pero que si podría tomar en cuenta para su ubicación física dentro del archivo. (Ibídem, p. 198 - 209, traducida por los autores).

Con lo que llevamos dicho hasta aquí sobre los procesos propuestos para la clasificación y ordenación de los archivos, podemos resumirlo de acuerdo a las consideraciones expuestas por Antoni M. Aragó, al referirse que la clasificación engloba tres actividades:

1. La actividad consistente en reconocer, definir y concretar los grandes grupos que forman el archivo (secciones, series, secuencias por materias y colecciones), considerando la morfología y la configuración prearchivística del fondo y diseñando el plan que se debe seguir provisionalmente para organizar el conjunto documental (estructuración general del archivo). 
2. La clasificación propiamente dicha, en el sentido concreto de constituir unas determinadas clases dentro del archivo, que pueden responder a una realidad o a una convención.

3. La operación de poner en orden (topográfico, cronológico, alfabético) las unidades dentro de las series o grupos constituidos. En realidad, se trata de la ordenación como complemento de la clasificación. (Aragó, 1979, citado en Alberch, 2003, p. 125)

\section{b) La valoración}

Puede considerarse que las formulaciones teóricas respecto al proceso de valoración en la Archivística clásica, hasta 1934, tienen un carácter incipiente, pues como apunta Malheriro (Ob. Cit) las preocupaciones por evaluar documentos archivísticos provienen del "...aumento considerable de la producción de documentos durante y después de la primera guerra mundial.” (p. 129, traducido por los autores).

Las posiciones respecto a la valoración en este período están marcadas por las ideas de archiveros ingleses y alemanes. En el caso alemán el criterio de la valoración está centrado en la preocupación de conservar los documentos para que sirvieran de fuentes para la investigación, al punto que Couture (1996) considera que los alemanes valoraban "...para conservar" a diferencia de que los ingleses valoraban "para eliminar" (p. 15, traducido por los autores).

En tal sentido la valoración se convirtió en Alemania en un problema central para la Archivística, al punto que el archivero Otto Muller fue pionero en la defensa de la “...necesidad de negar a las administraciones el derecho de ser los únicos jueces en materia de selección documental, pues los archivistas debían tener una influencia decisiva en la valoración." (Malheiro, Ob. Cit, p. 130, traducido por los autores).

Esta idea de Muller, se oponía claramente a las de Jenkinson, que pueden considerarse como dominantes en esta época en relación con la valoración. En tal sentido podemos decir que la valoración en la Inglaterra de estos años está claramente condicionada por las ideas de Jenkinson respecto al concepto de evidencia y a su carácter de imparcial e impoluta. Por tanto, en Inglaterra la tendencia fue a eliminar todos aquellos documentos que no tenían valor evidencial desde el punto de vista legal, histórico, estadístico, económico o cualquier otro uso oficial.

Sin embargo, la eliminación de los documentos para Jenkinson no podía realizarla el archivero, dada su condición de guardián de la evidencia, esta actividad la consigna, en palabras de Cook (Ob. Cit.):

\footnotetext{
....al creador de los documentos (...) y de este modo, [Jenkinson] hacía del Administrador el único agente para la selección y destrucción de sus propios documentos. Los archivistas, entonces, se harían cargo de los remanentes, exactamente de la misma manera en que lo habían hecho en su día con los documentos medievales y de principios de la era moderna, donde no era necesaria la destrucción en un ambiente de archivístico debido a las pequeñas acumulaciones. Mientras, que el mismo Jenkinson se preocupó porque estos Administradores podrían no destruir muchos documentos o destruir demasiados, o incluso crear documentos que podrían ser consecuentes con la historia o brindar evidencia imparcial de las transacciones, pero no presentó ninguna solución satisfactoria para estos dilemas. (p. 11, traducido por los autores).
}

La archivística clásica (es decir, la archivística como fue codificada por el Manual de Muller, Feith y Fruin de 1898) identifica como objeto el conjunto de documentos creados o recibidos por una administración o una 
oficina, e identifica el objeto físico como la entidad básica. Se considera que las interacciones entre las entidades básicas son orgánicas por naturaleza.

Los objetivos son: el control físico e intelectual de los documentos, en parte para la preparación de su publicación.

La metodología: consiste en la aplicación del principio de procedencia y el principio de orden natural.

Finalmente, la técnica puede ser caracterizada como la descripción formal de documentos físicos y su organización, no de acuerdo a su forma, sino de acuerdo a su clasificación natural, una clasificación que refleja la organización del creador de los documentos. (párr., 5, traducido por los autores).

\section{Conclusión}

La Archivística clásica (historicista, tecnisista, Como se puede observar en el proceso de valoración hay también una tendencia estatista, puesto que las preocupaciones por eliminar o conservar documentos están limitadas a las producciones documentales de los organismos estatales para realizar las transferencias a los archivos históricos, sin considerar en modo algunos documentos producidos por personas individuales o entidades privadas.

Resulta conveniente antes de finalizar este artículo observar el punto de vista de Thomassen respecto a la Archivística Clásica, ya que este autor resume de una forma muy precisa las características esenciales de esta ciencia que hemos venido desarrollando en el escrito. Thomassen (Ob. Cit.) considera que:

patrimonialista, culturalista) se caracteriza por tener como objeto de estudio el archivo histórico aparecido en Europa con posterioridad a la revolución francesa. En tal sentido el documento archivístico es visto como un objeto informativo físico, textual y fijo que sirve de fuente para la investigación histórica, por ser evidencia imparcial e impoluta de los hechos de sus creadores; y el archivero como el guardián de dicha evidencia.

Los procesos de tratamiento documental que pueden observarse en la Archivística clásica son la clasificación, enfocada en los aspectos orgánicos (oficina productora) del principio de procedencia, y la valoración, signada por los presupuestos extremos de valorar para eliminar documentos o para conservarlos.

\section{Bibliografía}

Alberch, R. (2003). Los Archivos, entre la memoria histórica y la sociedad del conocimiento. Barcelona: UOC.

(2011). Biografisch Woordenboek van Nederland:1880-2000 Historici.nl. Recuperado el 12 de Febrero de 2013 de http://www.historici.nl/Onderzoek/Projecten/BWN.

Casanova, E. (1928). Archivistica. Italia.: Lazzeri.

C.I.A. (1984). Dictionary of Archival Terminology. Mürichen, New York, London, Paris.

Cook, T. (1997). What is Past is Prologue: A History of Archival Ideas Since 1898, and the Future. Archivaria. № 43. pp. 17-63. Recuperado http://journals.sfu.ca/archivar/index.php/archivaria/article/view/12175/13184

Couture, C. (1996). L'évaluation des archives: Etat de la question. Archives. Vol 28. No.: 3-31.

(2011). Dizionario Biografico degli Italiani - A | Treccani, Vol. 21. (1978) Recuperado el 14 febrero, 2013, de http://www.treccani.it/lem

Duchein, M. (1992). The history of European archives and the development of the archival profession in Europe. American Archivist. Vol. 55. No. 1.: 14-25. Recuperado el 12 de febrero de 2013 de http://archivists.metapress.com/content/k17n44g856577888/

(2011). Dizionario Biografico degli Italiani - A | Treccani, Vol. 21. (1978) Recuperado el 14 febrero, 2013, de http://www.treccani.it/lem

Duranti, L. (1992, Sep.). Origin and Development of the Concept of Archival Description. Archivaria. Vol. 35.: 47-54. Recuperado el 26 de Febrero de 2013 de http://journals.sfu.ca/archivar/index.php/archivaria/article/viewArticle/11884

Duranti, L. (1993). The odyssey of records managers. Canadian archival studies and the rediscovery of provenance.: 29-60. 
Duranti, L. (1995). Archives as a place. Archives and Manuscripts. Vol. 24, No. 2.: 242-255.

Fuster, F. (1999). Archivística, Archivo, Documento de Archivo...Necesidad de Clarificar los Conceptos. ANALES DE DOCUMENTACIÓN. Norteamerica. Vol. No. 2. Consultado el 22 de febrero de 2013 de http://revistas.um.es/analesdoc/article/view/2631/2611

Gutiérrez, C., Lodolini, E., Tanodi, A. \& Gallego, O. (1991). Archivística. Lima: PUCP.

Heredia, A. (1991). Archivística general: teoría y práctica. (5ª. ed.) Sevilla: Excma. Diputación de Sevilla. 512

Horsman, P. Ketelaar, E. \& Thomassen, T. (2003 Otoño-Invierno). New Respect for the Old Order: The Context of the Dutch Manual. The American Archivist. Vol. 66. №2: 249-270. Recuperado el 12 de febrero de 2013 de http://archivists.metapress.com/content/452235k813568872/fulltext.pdf

Jekinson, H. (1922). Manual of archive administration: including the problems of war archives and archive making. Oxford: The Clarendon Press.

Martín-Pozuelo, M. (1996). La Construcción Teórica En Archivística: El Principio de Procedencia. Madrid: Universidad Carlos III de Madrid.

Moreno, A. (2004). El tiempo de la archivística: un estudio de sus espacios de racionalidad histórica. Ciência da Informação, Vol. 33. №3.: 76-96. Recuperado el 13 de febrero de 2013 de http://www.scielo.br/pdf/ci/v33n3/a10v33n3.pdf.

Muller, S., Feith, J. \& Fruin, R. (2003). Manual for the arrangement and description of archives. Society of Amer Archivists. (2ª ed.) Chicago: H.W. Wilson. (Original publicado en 1898).

(2010). NDB / EDB - Información histórico-biográfica en línea / Historisch. Recuperado el Febrero 12, 2013 de http://www.ndb.badw-muenchen.de/eb_lexika_es.htm.

Malheiro, A. (2002). Arquivística. Porto: Afrontamento.

(2009). II futuro della memoria. Atti del convegno internazionale - Archivi. Recuperado el 16 de Febrero de 2013 de http://www.archivi.beniculturali.it/serviziolll/pub/pas/saggi/sag-045.html.

Thomassen, T. (1999). The development of archival science and its european dimension: in The Archivist and the Archival Science. Seminar for Anna Christina Ulfsparre, (Lund: Landsarkivet, 1999). Recuperado el 10 de Febrero de 2013, del sitio Web de Zagreb Society Archives: http://daz.hr/zad/arhol/the-development-of-archival-science-and-its-european-dimension/

Wedgeworth, R. (Ed.). (1993). World Encyclopedia of library and information service. (3a . ed.). 


\section{Datos de los autores}

Leomar José Montilla Peña

Máster en Bibliotecología y Ciencias de la Información. Egresado de la Universidad de la Habana, Faculta de Comunicación, La Habana, Cuba. Licenciado en Bibliotecología y Ciencias de la Información por la misma universidad. Encargado de la Biblioteca Especializada del Instituto Nacional de Investigaciones Agrícola, Unidad Ejecutora Lara, Venezuela. Profesor Instructor del Programa Nacional de Formación en Ciencias de la Información de la Universidad Politécnica Territorial Andrés Eloy Blanco, Barquisimeto, Venezuela. Integrante del Comité Interinstitucional para el Fortalecimiento de la Gestión del Programa Nacional de Formación en Ciencias de la Información, Ministerio del Poder Popular para la Educación Universitaria, Caracas, Venezuela.

leomonp@gmail.com

\section{Mayra M. Mena Mujica}

Doctora en Ciencias de la Información por la Universidad de La Habana, Faculta de Comunicación, La Habana, Cuba. Máster en Bibliotecología y Ciencias de la Información y Licenciada en Historia por la misma universidad. Profesora Auxiliar del Departamento de Bibliotecología y Ciencias de la Información y profesora adjunta de la Facultad de Historia de la Universidad de La Habana. Miembro de la Comisión Nacional de la Carrera de Ciencias de la Información, de la Comisión de Grados Científicos y de la Comisión Técnica del Sistema Nacional de Archivos. Coordinadora Maestría en Bibliotecología y Ciencias de la Información, La Habana, Cuba.

mmena@infomed.sld.cu

$\begin{array}{ll}\text { Recibido-Received } & : 2013-07-10 \\ \text { Aceptado-Accepted } & : 2013-09-27\end{array}$

(c) $\mathbf{E Y}$ New articles in this journal are licensed under a Creative Commons Attribution 3.0 United States License.

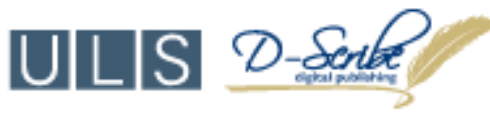

This journal is published by the University Library System of the University of Pittsburgh as part of its D-Scribe Digital Publishing Program and is cosponsored by the University of Pittsburgh Press. 